\title{
A pot of gold at the end of the cosmic "raynbow"?
}

\author{
L. A. Anchordoqui ${ }^{a *}$, M. T. Dova ${ }^{b \dagger}$, T. P. McCauley ${ }^{a \ddagger}$, T. Paul ${ }^{a \S}$, S. Reucroft ${ }^{a}$, and J. D. Swain ${ }^{a} \|$ \\ a Department of Physics, Northeastern University, Boston, MA 02115, USA \\ ${ }^{\text {b}}$ Departamento de Física, UNLP, CC 67 (1900) La Plata, Argentina
}

We critically review the common belief that ultrahigh energy cosmic rays are protons or atomic nuclei with masses not exceeding that of iron. We find that heavier nuclei are indeed possible, and discuss possible sources and acceleration mechanisms for such primaries. We also show detailed simulations of extensive air showers produced by "superheavy" nuclei, and discuss prospects for their detection in future experiments.

The unambiguous detection of cosmic rays (CRs) with energies above $10^{20} \mathrm{eV}$ (see [1] for a survey and bibliography on the subject) is a fact of outstanding astrophysical interest. As shown in the pioneering works of Greisen, Zatsepin, and Kuzmin [2], the possible sources and the accelerating mechanisms are constrained by the observed particle spectra due to the interaction with the universal radiation and magnetic fields on the way to the observer. The low flux of particles at the end of the spectrum (the typical rate of CRs above $10^{20} \mathrm{eV}$ is one event $/ \mathrm{km}^{2} /$ century) puts strong demands on the collection power of the experiments, such as can only be achieved by extended air shower detection arrays at ground level. This indirect method of detection bears a number of serious difficulties in determining the energy, mass and/or arrival direction of the primary particles. Astrophysical mechanisms to accelerate particles to energies of up to $10^{21-22} \mathrm{eV}$ have been identified, but they require exceptional sites [3]. Essentially, the mechanisms rely on a net transfer of macroscopic kinetic energy of moving magnetized plasma to individual charged particles due to repeated collisionless scatterings with randomly moving inhomogeneities of the turbulent magnetic field, or with shocks in the medium.

\footnotetext{
*doqui@hepmail.physics.neu.edu

$\dagger_{\text {dova@venus.fisica.unlp. edu.ar }}$

$\ddagger_{\text {mccauley@hepmail.physics.neu.edu }}$

\$tom.paul@hepmail.physics.neu.edu

I stephen.reucroft@cern.ch

"ljohn.swain@cern.ch
}

Since the high-energy cutoff of shock acceleration increases with the charge number of the nucleus $Z$, heavy ions would be nice candidates for ultra high-energy CRs. In this talk we review the current status of superheavy-nuclei as primaries of the cosmic radiation.

It has been generally thought that ${ }^{56} \mathrm{Fe}$ is a significant end product of stellar evolution and higher mass nuclei are rare in the cosmic radiation. Strictly speaking, the atomic abundances of middle-weight $(60 \leq A<100)$ and heavy-weight $(A>100)$ elements are approximately 3 and 5 orders of magnitude lower, respectively, than that of the iron group [4]. The explosion of the H-rich envelopes of type II supernovae has long been held responsible for the synthesis of the stable superheavy nuclides. There is observational evidence that these nuclides are synthesized in a variety of chemically peculiar Red Giants [5] and in special objects like FG Sagittae [6] or SN1987 A [7]. Consequently, starbursts (astrophysical environments which comprise a considerable population of $\mathrm{O}$ and Red Giant stars [8], with a supernovae rate as high as $0.2-0.3 \mathrm{yr}^{-1}$ [9]) appear as the natural sources able to produce relativistic super-heavy nuclei. Very recently, we have presented a comprehensive study of a possible nearby superheavynucleus-Zevatron [10]. We have shown that it is likely that nuclei heavier than iron with energies above a few $\mathrm{PeV}$ can escape from the dense core of a nearby starburst galaxy (like M82, NGC 253), and eventually be re-accelerated to superhigh energies $\left(E \geq 10^{20} \mathrm{eV}\right)$ at the terminal shocks of 
galactic superwinds generated by the starburst. ${ }^{7}$ Specifically, ions are diffusively accelerated up to a few $\mathrm{PeV}$ at single supernova shock waves in the nuclear region of the galaxy without suffering significant spallation reactions [12]. The cosmic ray outflow is convection dominated, and the typical residence time of the nuclei in the starburst results in $t \sim 1 \times 10^{11} \mathrm{~s}$. Thus, the total path traveled is substantially shorter than the mean free path (which scales as $A^{-2 / 3}$ ) of a super-heavy nucleus (for details see [13]). Those which are able to escape from the central region without suffering catastrophic interactions could be eventually re-accelerated to superhigh energies at the terminal shocks of galactic superwinds generated by the starburst. In terms of parameters that can be determined from observations the maximum energy of the nuclei reads (again the reader is referred to [13]),

$E_{\max } \approx \frac{1}{2} Z e B \frac{\dot{E}_{\mathrm{sw}}}{\dot{M}} \tau$.

The predicted kinetic energy and mass fluxes of the starburst of NGC 253 derived from the measured IR luminosity are $\dot{E}_{r m s w} \approx 2 \times 10^{42} \mathrm{erg} \mathrm{s}^{-1}$ and $\dot{M} \approx 1.2 \mathrm{M}_{\odot} \mathrm{yr}^{-1}$, respectively [14]. The starburst age is estimated from numerical models that use theoretical evolutionary tracks for individual stars and make sums over the entire stellar population at each time in order to produce the galaxy luminosity as a function of time [15]. Fitting the observational data these models provide a range of suitable ages for the starburst phase that, in the case of NGC 253, goes from $5 \times 10^{7}$ to $1.6 \times 10^{8} \mathrm{yr}$ (also valid for M82) [15]. These models must assume a given initial mass function (IMF), which usually is taken to be a powerlaw with a variety of slopes. Recent studies have shown that the same IMF can account for the properties of both NGC 253 and M82 [16]. Here we shall assume a conservative age $\tau=50 \mathrm{Myr}$. Finally, the radio and $\gamma$-ray emission from NGC 253 are well matched by models with $B \sim 50 \mu \mathrm{G}$ [17]. All in all, the balance between the energy gains and synchrotron/photodisintegration losses

\footnotetext{
${ }^{7}$ It is important to stress that M82 is positioned close to the arrival direction of the highest CR event detected on Earth. This was first pointed out in [11].
}

[18] of a super-heavy nucleus (like gold) leads to a maximum energy of a few hundred $\mathrm{EeV}{ }^{8}$

Let us turn now to the analysis of the energy loses during propagation. The energetic nucleus is seen to lose energy mainly as a result of its photodisintegration. In the universal rest frame (in which the microwave backgroud radiation is at $3 K$ ), the disintegration rate $R$ of an extremely high energy nucleus with Lorentz factor $\Gamma$, propagating through an isotropic soft photon background of density $n$ is given by [19],

$R=\frac{1}{2 \Gamma^{2}} \int_{0}^{\infty} d E \frac{n(E)}{E^{2}} \int_{0}^{2 \Gamma E} d E^{\prime} E^{\prime} \sigma\left(E^{\prime}\right)$,

where primed quantities refer to the rest frame of the nucleus, and $\sigma$ stands for the total photon absortion cross section. Above $10^{20} \mathrm{eV}$, the energy losses are dominated by collisions with the relic photons. The fractional energy loss around this energy is $R \sim 10^{-15} \mathrm{~s}^{-1}$. With this in mind, it is straightforward to check that superheavy nuclei may reach the Earth (for details see Fig. 2 of Ref. [10]). In the rest of this report we shall discuss the characteristics of the extensive air showers that these nuclei may produce after interaction with the atmosphere.

Golden Shower Simulations: In order to perform the simulations we shall adopt the superposition model. This model assumes that an average shower produced by a nucleus with energy $E$ and mass number $A$ is indistinguishable from a superposition of $A$ proton showers, each with energy $E / A$. We have generated several sets of ${ }^{197} \mathrm{Au}$ air shower sinulations by means of the AIREs Monte Carlo code [20]. The sample was distributed in the energy range of $10^{18}$ up to $10^{20.5} \mathrm{eV}$. SIBYLL was used to reproduce hadronic collisions above $200 \mathrm{GeV}$ [22]. All shower particles with energies above the following thresholds were tracked: $750 \mathrm{keV}$ for gammas, $900 \mathrm{keV}$ for electrons and positrons, $10 \mathrm{MeV}$ for muons, $60 \mathrm{MeV}$ for mesons and $120 \mathrm{MeV}$ for nucleons and nuclei. The particles were injected vertically at the top of the atmosphere (100 km.a.s.l), and the surface de-

\footnotetext{
${ }^{8}$ It should be stressed that for $A>140$ the bulk solarsystem abundance distribution peaks at $A=195$ [21]. To make some estimates, we then refer our calculations to a gold nucleus.
} 
tector array was put at a depth of $1036 \mathrm{~g} / \mathrm{cm}^{2}$, i.e., at sea level. Secondary particles of different types and all charged particles in individual showers were sorted according to their distance $R$ from the shower axis.

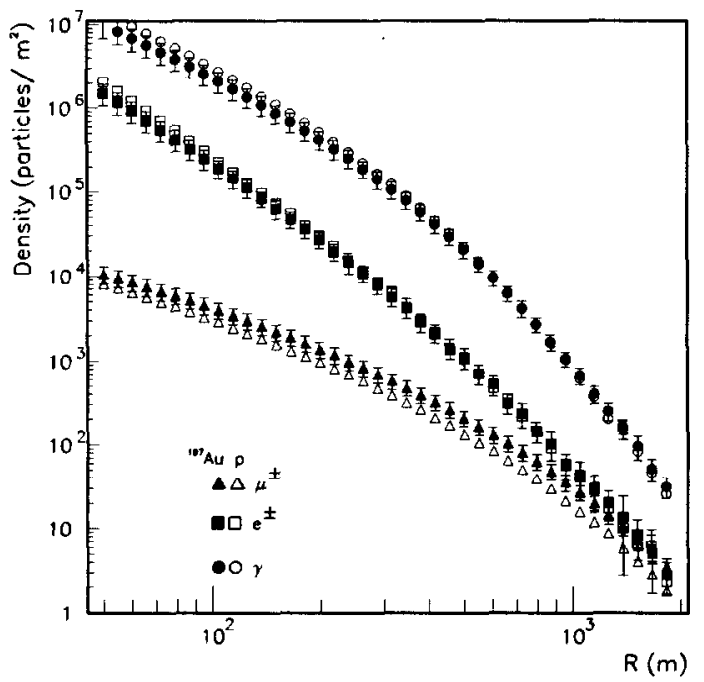

Figure 1. Ground lateral distributions of proton and ${ }^{197} \mathrm{Au}$ air-showers. The incident energy is $E=3 \times 10^{20} \mathrm{eV}$.

In Fig. 1 we show the lateral distributions of different groups of secondary particles (we have considered separately $\gamma, e^{+} e^{-}$, and $\mu^{+} \mu^{-}$). One can see that the number of muons from the gold nucleus shower is greater than the number of muons from the proton shower.

As the cascade develops in the atmosphere, it grows until a maximum size (number of particles) is reached. The location in the atmosphere where the cascade has developed the maximum size is denoted by $X_{\max }$, with units of $\mathrm{g} \mathrm{cm}^{-2}$. For cascades of a given total energy, heavier nuclei have smaller $X_{\max }$ than nucleons because the shower is already subdivided into $A$ nucleons when it enters the atmosphere. At $10^{20} \mathrm{eV}$, the $\left\langle X_{\max }\right\rangle$ of a proton (gold) shower is $\approx 879 \mathrm{~g} / \mathrm{cm}^{2}(\approx 777$ $\left.\mathrm{g} / \mathrm{cm}^{2}\right)$. A dust-grain has an even larger cross section, so it tends to interact sooner than protons and nuclei [24]. In Fig. 2, we compare the longitudinal profile of showers initiated by a proton, a gold-nucleus and a dust-grain. It is clearly seen how the $X_{\max }$ decreases when increasing the mass. The simulated gold shower is partially consistent with the Fly's Eye data. Furthermore, its longitudinal development better reproduces the data than protons or dust-grains. It should be remarked, however, that extensive air shower simulation depends on the hadronic interaction model [25]. We also point out that for the simulation, detector effects were not taken into account.

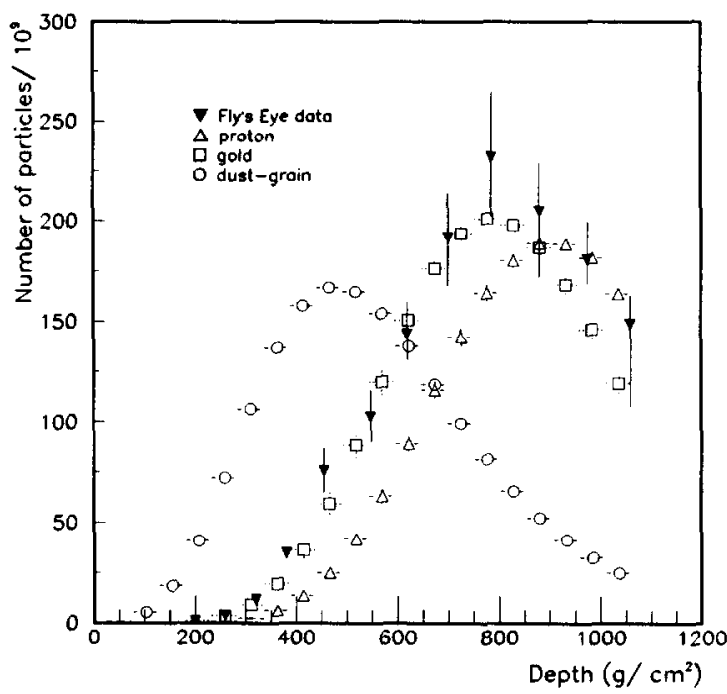

Figure 2. Longitudinal development of $3 \times 10^{20}$ $\mathrm{eV}$ showers induced by a proton, a gold-nucleus and a dust-grain $(\log \Gamma=4.5)$, together with the data of the highest event recorded by Fly's Eye [23].

Even though the superheavy nucleus hypothesis is partially supported by data from the CASAMIA experiment [26], more data is certainly needed to verify this model. In order to significantly increase the statistics at the end of 
the spectrum, the Southern Auger Observatory is currently under construction [27]. It will consist of a surface array which will record the lateral and temporal distribution of shower particles, and an optical air fluorescence detector, which will observe the air shower development in the atmosphere. These two techniques provide complementary methods of extracting the required information from the shower to test the ideas discussed in this paper.

Note added in proof: After this talk was presented we have completed the air shower analysis by using a more inelastic hadronic model for the first generation of particles [28]. Definite conclusions on the nature of the highest energy Fly's Eye event cannot be reached, mainly because of large fluctuations from model to model. However, it should be stressed that even though the primary chemical composition remains hidden by the hadronic interaction model, it is evident that in both cases the shower profile is inconsistent with a proton, or a gamma-ray primary.

This work was partially supported by CONICET, Fundación Antorchas and the National Science Foundation.

\section{REFERENCES}

1. S. Yoshida, and H. Dai, J. Phys. G 24 (1998) 905.

2. K. Greisen, Phys. Rev. Lett. 16 (1966) 748; G. T. Zatsepin, and V. A. Kuzmin, Pis'ma 7h. Fksp. Teor. Fiz. 4 (1966) 114.

3. P. Bhattacharjee and G. Sigl, Phys. Rep. 327, 109 (2000) 109, and references therein.

4. E. M. Burbidge, G. R. Burbidge, W. A. Fowler, and F. Hoyle, Rev. Mod. Phys. 29 (1957) 547.

5. V. V. Smith, in Cosmic Abundances of matter, AIP Conference Proceedings 183, ed. C. J. Waddington (New York, AIP 1989), p. 200.

6. G. E. Langer, R. P. Kraft, K. S. Anderson, Astrophys. J. 189 (1974) 509.

7. P. A. Mazzali, L. B. Lucy, K. Butler, Astron. Astrophys. 258 (1992) 399.

8. S. Sakai and B. Madore, Detection of the Red Giant Branch Stars in M82 Using the Hubble
Space Telescope, [astro-ph/9906484].

9. See for instance, T. W. B. Muxlow et al., Mont. Not. Roc. Astron. Soc. 266 (1994) 455; J. S. Ulvestad and R. J. Antonucci, Astrophys. J. 448 (1997) 621; D. A. Forbes et al., Astrophys. J. 406 (1993) L11; R. de Grijs et al., Supernova Remnants in the Fossil Starburt in M82, [astro-ph/9909044].

10. L. A. Anchordoqui, M. T. Dova, T. P. McCauley, S. Reucroft and J. D. Swain, Phys. Lett. B 482 (2000) 343.

11. J. W. Elbert and P. Sommers, Astrophys. J. 441 (1995) 151.

12. P. O. Lagage and C. J. Cesarsky, Astron. Astrophys. 125 (1983) 249.

13. L. A. Anchordoqui, G. E. Romero, and J. A. Combi, Phys. Rev. D 60 (1999) 10300.

14. T. M. Heckman, L. Armus, and G. K. Miley, Ap. J. Suppl. 74 (1990) 833.

15. G. H. Rieke, et al., Ap. J. 238 (1980) 24.

16. C. W. Engelbracht, et al., Ap. J. 505 (1998) 639.

17. T. A. D. Paglione, A. P. Marscher, J. M. Jackson, and D. L. Bertsch, Ap. J. 460 (1996) 295.

18. R. J. Protheroe, [astro-ph/9812055].

19. F. W. Stecker, Phys. Rev. D 180 (1969) 1264.

20. S. J. Sciutto, in Proc. XXVI International Cosmic Ray Conference, Edts. D. Kieda, M. Salamon, and B. Dingus, Salt Lake City, Utah, 1999) vol.1, p.411.

21. E. Anders and N. Grevesse, Geochim. Cosmochim. Acta 53 (1989) 197.

22. R. S. Fletcher, T. K. Gaisser, P. Lipari and T. Stanev, Phys. Rev. D 50 (1994) 5710.

23. D. J. Bird et al., Astrophys. J. 441 (1995) 144.

24. L. A. Anchordoqui, Phys. Rev. D 61 (2000) 087302.

25. L. A. Anchordoqui, M. T. Dova, L. N. Epele and S. J. Sciutto, Phys. Rev. D 59 (1999) 094003.

26. M. A. K. Glasmacher et al., Astropart. Phys. 12 (1999) 1.

27. http://www.auger.org

28. L. A. Anchordoqui, M. Kirasirova, T. P. McCauley, S. Reucroft and J. Swain, Phys. Lett. B 492 (2000) 237. 